\title{
Return to work journey: an injured worker perspective
}

Peta Odgers

Comcare, Canberra ACT 2600, Australia. peta.odgers@comcare.gov.au

Background: Returning to work following a workplace injury or illness is a complex, multi-faceted and continuously evolving issue that involves a range of challenges and complexities. Very little, however, is known about the stages individual injured worker's progress through, from their personal perspective, in returning to work.

Objectives: The objectives of this research were to provide Comcare with greater insights and a better understanding about how to positively engage with injured/unwell workers in the federal jurisdiction, and to encourage more positive return to work (RTW) and recovery outcomes.

Methods: A total of 47 in-depth interviews were conducted with injured workers from both premium paying and self-insured organisations in four Australian States and Territories. An independent research company conducted the interviews on behalf of Comcare between December 2012 and February 2013. A purposeful sampling approach was employed, however, this was influenced by the willingness of people to participate. Principle Findings:- Six stages were identified in the RTW journey, with each stage representing a distinct dynamic. The mind-set and needs of an individual were found to shift through each of these stages, thereby influencing the likelihood of them achieving a successful RTW outcome.

Discussion: The RTW experience of injured and unwell workers was found to be a very personal journey that encompasses a range of experiences which can be completed in a few weeks, or continue for years. It is very emotionally charged, challenging, and tends to result in changes to personal and professional identity, life and work expectations.

Conclusion: The insights gained by Comcare into an individual's RTW journey have reaffirmed our commitment to having the injured/unwell worker at the centre of what we do, and to continuing to tailor our claims management strategies to meet their individual needs. le: Key factors influencing an organisation's approach to return to work. 